\title{
THE USE OF CONTINUUM THERMODYNAMICS IN THE FORMULATION OF ICE-SHEET DYNAMICS
}

\author{
by \\ K. Hutter \\ (Fachbereich 6, Mechanik, Technische Hochschule, Hochschulstraße 1, D-6100 Darmstadt, \\ Federal Republic of Germany) \\ and \\ H. Engelhardt
}

(Versuchsanstalt für Wasserbau, Hydrologie und Glaziologie, ETH-Zentrum,

Gloriastraße 37-39, CH-8092 Zürich, Switzerland)

\section{ABSTRACT}

Ice sheets consist of several disjoint regions, each with physically distinct behavior. Parts are cold; others are temperate and either partly or completely saturated. Varying dust content, impurities, debris content, etc., may affect the ice flow. Usually, these regions are separated by material or non-material surfaces or boundaries. We use mixture concepts, involving the balances of mass, momentum, energy and entropy. When applied to regular domain points these concepts and appropriate constitutive postulates yield the field equations for the evolution of the constituents. When formulated in terms of singular surfaces, boundary and transition conditions emerge. Our presentation takes the form of an extended abstract of work that is presently under consideration (Hutter and Engelhardt 1988; Hutter and Engelhardt, in preparation).

\section{DEFINITIONS AND NOTATION}

\section{Mixture quantities:}

Mixture (barycentric) velocity: $v^{i}=\frac{1}{\rho} \sum_{\alpha} \rho_{\alpha} v_{\alpha}^{i}$

Diffusion velocity: $u_{\alpha}^{i}=v_{\alpha}^{i}-v^{i}$

Mixture mass density: $\rho=\sum_{\alpha} \rho_{\alpha}$

Mixture stress: $t^{i j}=\sum_{\alpha}\left[t_{\alpha}^{i j}-\rho_{\alpha} u_{\alpha}^{i} u_{\alpha}^{j}\right]$

Mixture body force: $\rho f^{i}=\sum_{\alpha} \rho_{\alpha} f_{\alpha}^{i}$

Mixture internal energy density:

$$
\rho \varepsilon=\sum_{\alpha} \rho_{\alpha}\left[\varepsilon_{\alpha}+\frac{1}{2} u_{\alpha}^{i} u_{\alpha i}\right]
$$

Mixture heat flux:

$$
q^{i}=\sum_{\alpha}\left[q_{\alpha}^{i}+\rho_{\alpha}\left(\varepsilon_{\alpha}+\frac{1}{2} u_{\alpha}^{k} u_{\alpha k}\right) u_{\alpha}^{i}-t_{\alpha}^{i j} u_{\alpha j}\right]
$$

Mixture internal energy supply density:

$$
\rho r=\sum_{\alpha} \rho_{\alpha}\left(r_{\alpha}+\frac{1}{2} f_{\alpha}^{i} u_{\alpha i}\right)
$$

Mixture entropy density: $\rho \eta=\sum_{\alpha} \rho_{\alpha} n_{\alpha}$

Mixture entropy density supply: $\rho s=\sum_{\alpha} \rho_{\alpha} s_{\alpha}$

Mixture entropy density production: $\rho \gamma=\sum_{\alpha} \rho_{\alpha} \gamma_{\alpha}$

Mixture entropy density flux: $\phi^{i}=\sum_{\alpha}\left[\phi_{\alpha}^{i}-\rho_{\alpha} \eta_{\alpha} u_{\alpha}^{i}\right]$

\section{Constituent quantities:}

$\mathrm{c}_{\alpha}$ Rate of mass production of constituent $\alpha$

$c_{\alpha}$ Specific heat of constituent $\alpha$

$D_{\alpha}^{i j}$ Components of the stretching tensor of constituent $\alpha$

$f_{\alpha}^{i}$ Body force components

$g^{i}$ Components of gravity

$\ell_{\alpha}$ Rate of energy production

$m_{\alpha}^{i}$ Momentum production rate components

$n_{\alpha}$ Volume fraction

$p_{\alpha}$ Pressure

$q_{\alpha}^{i}$ Heat flux components

$r_{\alpha}$ Energy supply

$s_{\alpha}$ Entropy supply

$T_{\alpha}$ Temperature

$t_{\alpha}^{i j}$ Partial Cauchy stress tensor components

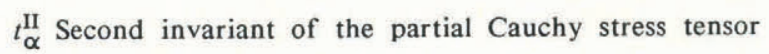

$v_{\alpha}^{i}$ Material velocity components

$\gamma_{\alpha}$ Specific entropy production rate

$\varepsilon_{\alpha}$ Specific internal energy

$n_{\alpha}$ Specific entropy

$\rho_{\alpha}$ Partial density

$\hat{\rho}_{\alpha}$ True density

$\phi_{\alpha}^{i}$ Entropy flux

Other quantities:
$A(\cdot)$ Rate factors
$a_{\perp}^{c}$ Melting/freezing rate at cold-temperate transition 
$\hat{e}^{i}$ Components of the unit vector perpendicular to the singular surface

\section{$f\left(t^{\mathrm{II}}\right)$ Creep response function}

$k$ Darcy's permeability

$L \quad$ Latent heat of fusion

$t$ Time

$v_{\mathrm{F}}^{i}$ Seepage velocity components

$x^{i} x, y, z$ Position components and coordinates

$z_{\mathrm{c}}(x, y, t) \quad z$-coordinate defining cold-temperate transition surface

$w^{i}$ Singular surface velocity components

$\lambda$ Density ratio $=\hat{\rho}_{i} / \hat{\rho}_{w}$

$\Phi$ Gravitational potential

\section{INTRODUCTION}

Ice sheets consist of several disjoint regions, each with physically distinct behavior. Parts are cold; others are temperate and either partly saturated or completely saturated with water. Varying dust content, impurities, grain-size and crystal orientation may affect the ice flow. At depth, gravel may be dispersed within the ice and affect the mechanical properties of the pre-basal layer. Sub-basal conditions may be dominated by sliding over hard rock or by the deformation of water-saturated sediments. Water pressure in the sub-sole drift turns out to play a decisive role. Of a special nature is the sub-sole water drainage system, which may exhibit distinct modes of water flow, with different flow characteristics. A thick sub-sole deformable sediment bed is permeable to water flow, and a Darcy-type water-flow regime may be applicable. Generally, the transition from one kind of behavior to the other is gradual, but transition times are often small, so that an abrupt change may be a reasonable approximation. The various domains are then separated by material and non-material surfaces where some of the field variables may suffer finite jump discontinuities. Such surfaces are the free surface, the transition surface between cold and temperate ice, the phreatic surface, the ice-ocean, the ice-sediment and the sediment-rock interfaces.
Figure 1 illustrates how the situation may realistically arise. The entire grounded ice sheet consists of the following separate regions:

(1) Cold ice

Temperatures are below freezing and vary according to the amount of heat that is advected and conducted.

\section{(2a) Saturated temperate ice}

Ice and water coexist in a proportion dictated by influx from above, discharge to the ground, and strain heating due to shearing and compression. Pores, veins and cavities are completely filled with water.

(2b) Non-saturated temperate ice

The conditions are the same as in (2a), except that there is not enough water available to fill the "pore volume" completely. Percolation may be a decisive process that governs the water flow through the pores.

\section{(3a) Debris-laden cold ice}

This is a relatively thin layer, where ice and dispersed sediments coexist. Sometimes this layer is only a few meters thick.

(3b) Debris-laden temperate ice

This is a layer up to a few meters thick, where ice, water and rock granules coexist.

\section{(4a) Water-filled sediment}

This layer exists because the temperate debris-laden ice above it melts at the contact surface and thus loses gravel to layer 4a. As the water has access to this layer, its mechanical properties depend upon the amount of water present. This layer is usually only a few meters thick.

\section{(4b) Abrasion zone}

Here frictional processes dominate. Till is abraded from the hard bedrock and transported along the base to form the soft deformable bed in the down-stream layer, $4 \mathrm{a}$.

This picture is not complete, as it ignores hard-bed sub-sole water drainage with cavity formation. We ignore it here in order to keep the picture as simple as possible. Furthermore, each domain is separated from any other by a surface, which may actually be a region, a thin transition or boundary layer and not as well defined as a surface. For clarity, however, we prefer the concept of a singular surface.

It may be unreasonable, or even unnecessary, to describe mathematically the full system in all its complexity

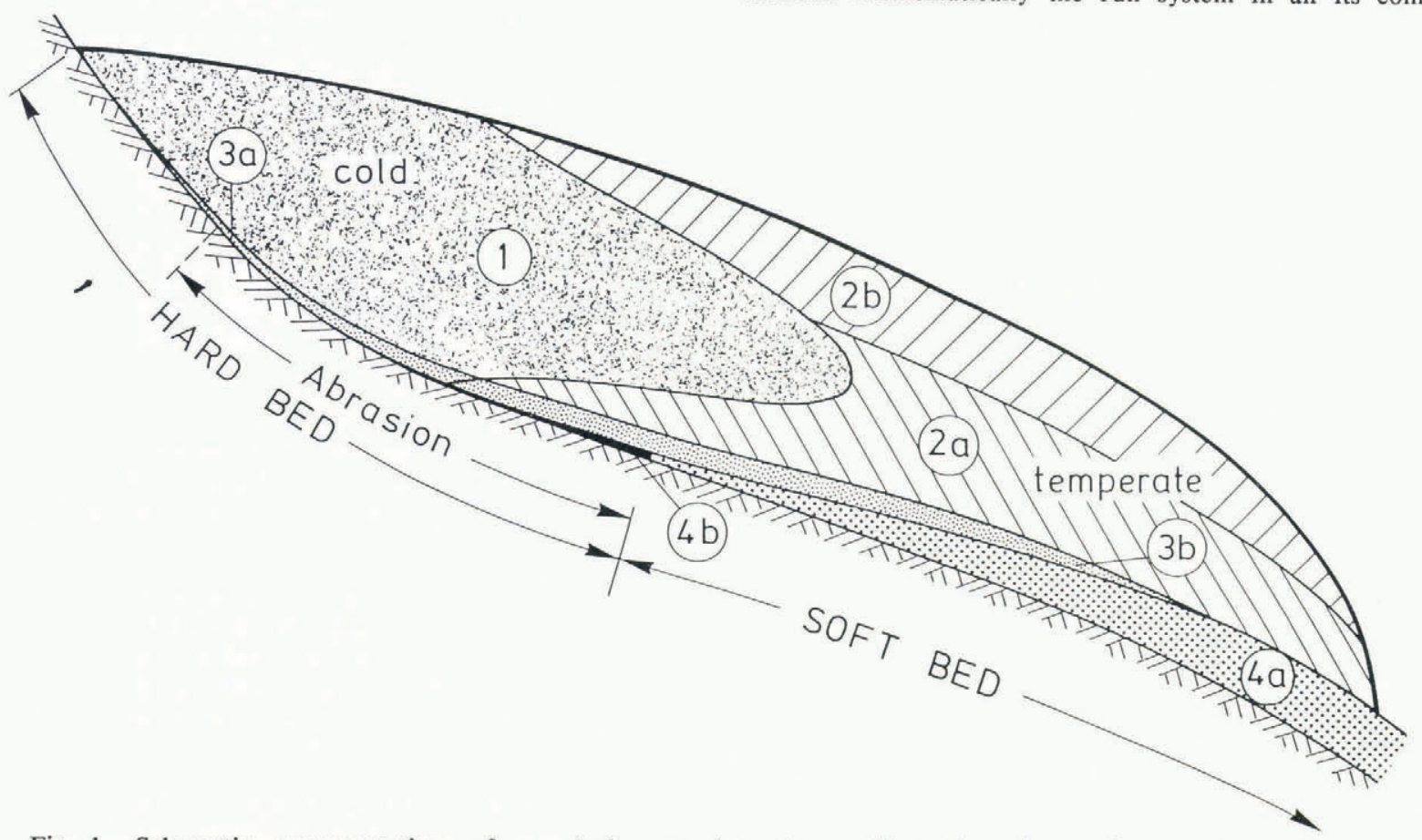

Fig. 1. Schematic representation of a glacier or ice sheet, illustrating its various regions. For explanations, see main text. 
as illustrated in Figure 1. To make things simpler, we consider interesting parts of this system as separate from the rest. For instance, interpretation of temperature data in a bore hole drilled through polythermal ice (upper layer cold, lower layer temperate) requires a clear understanding of the momentum and energy balances in the cold and temperate parts of the hole, including the processes at the cold-temperate transition surface with its kink in the temperature profile.

\section{TABLE Ia. MIXTURE-FIELD RELATIONS}

Mass density $=\rho$ :

Constituent $\alpha$ :

$$
\frac{\partial \rho_{\alpha}}{\partial t}+\left(\rho_{\alpha} v_{\alpha}^{i}\right), i=c_{\alpha}
$$

Mixture:

$$
\frac{\partial \rho}{\partial t}+\left(\rho v^{i}\right)_{i}=0
$$

Balance:

$$
\sum_{\alpha} c_{\alpha}=0
$$

Linear momentum density $=\rho \mathbf{v}$ :

Constituent $\alpha$ :

$$
\frac{\partial\left(\rho_{\alpha} v_{\alpha}^{i}\right)}{\partial t}+\left(\rho_{\alpha} v_{\alpha}^{i} v_{\alpha}^{j}-t_{\alpha}^{i j}\right),{ }_{j}-\rho_{\alpha} f_{\alpha}^{i}=m_{\alpha}^{i}
$$

Mixture:

$$
\frac{\partial\left(\rho v^{i}\right)}{\partial t}+\left(\rho v^{i} v^{j}-t^{i j}\right), j-\rho f^{i}=0
$$

Balance:

$$
\sum_{\alpha} m_{\alpha}^{i}=0
$$

Total energy density $=\varepsilon \equiv \rho\left[\varepsilon+\frac{1}{2} v^{i} v_{i}\right]$ :

Constituent $\alpha$ :

$$
\frac{\partial \varepsilon_{\alpha}}{\partial t}+\left[\varepsilon_{\alpha} v_{\alpha}^{i}+\left(q_{\alpha}^{i}-t_{\alpha}^{i j} v_{\alpha j}\right){ }_{i}-\rho_{\alpha} r_{\alpha}-\rho_{\alpha} f_{\alpha}^{i} v_{\alpha i}=\ell \alpha\right.
$$

Mixture:

$$
\frac{\partial \varepsilon}{\partial t}+\left[\varepsilon v^{i}+\left(q^{i}-t^{i j} v_{j}\right)\right]_{, i}-\rho r-\rho f^{i} v_{i}=0
$$

Balance:

$$
\sum_{\alpha} l_{\alpha}=0
$$

Entropy density $=\rho n$ :

Constituent $\alpha$ :

$$
\frac{\partial\left(\rho_{\alpha} n_{\alpha}\right)}{\partial t}+\left(\rho_{\alpha} n_{\alpha} v_{\alpha}^{i}-\phi_{\alpha}^{i}\right)_{i}-\rho_{\alpha} s_{\alpha}=\rho_{\alpha} \gamma_{\alpha}
$$

Mixture:

$$
\frac{\partial(\rho \eta)}{\partial t}+\left(\rho \eta v^{i}-\phi^{i}\right)_{{ }_{i}}=\rho s=\rho \gamma
$$

Balance:

$$
\sum_{\alpha} \rho_{\alpha} \gamma_{\alpha}=\rho \gamma \geqslant 0
$$

Similarly, if one wants to model the formation of the sediment bed, a detailed description of the abrasion mechanism at the cold bed is required; the motion of the debris into the ice, and its advection through and with the ice, must be described, and the deposition mechanism in the temperate debris-laden ice must be modeled.

The formulation of these problems is simplified in the context of continuum thermodynamics. Each domain is regarded as a continuum, and the physical behavior is

TABLE Ib. CONSTITUENT JUMP CONDITIONS

$$
[f] \equiv f^{+}-f^{-}
$$

Mass:

$$
\left[\rho_{\alpha}\left(v_{\alpha}^{i}-w_{\alpha}^{i}\right) e_{i}\right]=0
$$

Linear momentum:

$$
\left[\rho_{\alpha} v_{\alpha}^{i}\left[v_{\alpha}^{j}-w_{\alpha}^{j}\right]-t_{\alpha}^{i j}\right] e_{j}=0
$$

Energy:

$$
\left[\varepsilon_{\alpha}\left[v_{\alpha}^{i}-w_{\alpha}^{i}\right]+\left[\begin{array}{ll}
a_{\alpha}^{i}-t_{\alpha}^{i j} & v_{\alpha j} j
\end{array}\right] e_{i}=0\right.
$$

Entropy:

$$
\left[\rho_{\alpha} n_{\alpha}\left[v_{\alpha}^{i}-w_{\alpha}^{i}\right]+\phi_{\alpha}^{i}\right] e_{i}=0
$$

described by formulating two or three constituent mixture concepts involving the balances of mass, momentum, energy and entropy. When applied to regular domain points, these concepts and appropriate constitutive postulates yield the field equations for ice and water flow, temperature distribution and for salt and dust transport. Alternatively, applying these concepts to singular surfaces (the transition surfaces) permits deduction of boundary and transition conditions. Sub-sets of equations describe simpler ice-sheet and glacier-flow problems known in the literature.

\section{A SELECTION OF EQUATIONS}

The governing equations involve statements that are common to all materials and form the foundation for all problems of continuum physics and statements that distinguish one material from the other. The former comprise the balance laws of mass, momentum, energy and entropy. Within a region where no discontinuities are encountered, they yield mixture-field relations (Table Ia). On interfaces these laws lead to the corresponding jump conditions (Table Ib). The laws which distinguish the materials comprise the constitutive relations.

The distinctive features of mixture concepts are:

(I) Mass, momentum and energy of the individual components may not be conserved, as there can be mass, momentum and energy production. The summed balances of mass, momentum and energy must, however, form conservation laws. This imposes restrictions on the production terms.

\section{Example 1}

Consider temperate ice as a mixture of ice and water. The melting rate is a local source of water and correspondingly a sink of ice. The sum adds up to zero.

\section{Example 2}

In temperate saturated ice the water forming the moisture content moves through the ice much like ground water. The force exerted by the water on the ice is equilibrated by an equal and opposite force exerted by the ice on the water. The mathematical formulation of this process leads essentially to Darcy's law.

(II) The variables describing the evolution of the mixture as a whole and those of each constituent are related to each other. For instance, 


$$
\begin{gathered}
\rho=\sum_{\alpha} \rho_{\alpha} \\
v^{i}=\frac{1}{\rho} \sum \rho_{\alpha} v_{\alpha}^{i} \\
t^{i j}=\sum_{\alpha}\left[t_{\alpha}^{i j}-\rho_{\alpha} u_{\alpha}^{i} u_{\alpha}^{j}\right] \\
q^{i}=\sum_{\alpha}\left[q_{a}^{i}+\rho_{\alpha}\left[\varepsilon_{\alpha}+\frac{1}{2} u_{\alpha}^{2}\right] u_{\alpha}^{i}-t_{\alpha}^{i j} u_{\alpha j}\right]
\end{gathered}
$$

where

$$
u_{\alpha}^{i}=v_{\alpha}^{i}-v^{i}
$$

Definitions and notation are given at the beginning of the paper.

\section{Example 3}

Consider temperate saturated ice and assume that we ignore the small volume changes in the mixture. Then $\rho$ is constant and known. This loss of a field variable must be compensated for by the introduction of another variable, uşually the pressure $p$. So we write $t^{i j}=$ $-p \delta^{i j}+t^{i j}$, where $t^{i j}$ is now the part of the stress that must be determined by constitutive assumptions. How is this pressure distributed among the stresses $t^{i j}$ ? There is no unique way to do this, but relation (2) must be obeyed. One way is to write

$$
t_{\alpha}^{i j}=-\frac{\rho_{\alpha}}{\rho} p \delta^{i j}+t_{\alpha}^{i j^{\prime}}
$$

A second way is provided by

$$
t_{\alpha}^{i j}=-n_{\alpha} p \delta^{i j}+t_{\alpha}^{i j^{\prime}}
$$

where $n_{\alpha}$ is the volume fraction of the constituent. Note that $\sum_{\alpha} n_{\alpha}=1$. Both relations satisfy condition (2);

Equation (4) is based on mass fraction, $x_{\alpha}=\rho_{\alpha} / \rho$, and Equation (5) is based on volume fraction.

(III) Equation (2) relates the mixture stresses and mixture heat flux to those of the constituents plus further quantities. When postulating material behavior, statements like relation (2) must be borne in mind. In other words, constitutive relations for the mixture quantities must be consistent with those of the components.

\section{Example 4}

Consider the water-filled sediment layer below the temperate ice region (4a in Fig. 1). Assume that both water and sediment as individual bodies conduct heat according to Fourier-type heat-flux relationships,

$$
q_{\alpha}^{i}=-k_{\alpha} \delta^{i j} T_{\alpha, j}
$$

Then the second part of Equation (2) directly implies that in a theory that gives one single temperature, $T=T_{\alpha}$, for all $\alpha$, Fourier's heat law cannot hold for the mixture as a whole.

(IV) It goes almost without saying that constitutive relations must be objective and obey the second law of thermodynamics.

\section{Example 5}

Consider saturated temperate ice that is modeled as a mixture of ice and water. It is known from continuum mechanics that the constituent momentum $m_{\alpha}$ is not an objective quantity and thus cannot, in general, be used as a constitutive quantity. The appropriate quantity is given in component form by

$$
m_{\alpha}^{i}+\mathrm{c}_{\alpha} v_{\alpha}^{i}\left[ \pm f\left(\mathrm{c}_{\alpha}\right) u_{\alpha}^{i}\right]
$$

where $c_{\alpha}$ is the mass-production rate of constituent $\alpha$. As a consequence, Darcy's law cannot blindly be taken over for temperate ice in order to model the water flow. The objective alternative to Darcy's law is

$$
m_{\mathrm{w}}^{i}=\frac{\mu}{k^{*}} n_{\mathrm{w}}\left(v_{\mathrm{w}}^{i}-v_{\mathrm{i}}^{i}\right)-\mathrm{c}_{\mathrm{w}} v_{\mathrm{w}}^{i}
$$

where $\mu$ is the viscosity of water $(0.1 \mathrm{Pas}), k^{*}$ is the coefficient of absolute permeability of the ice, $n_{w}$ is the porosity and $c_{w}$ is the rate of water production per unit volume. Clearly, for $c_{w}=0$, Equation (6) reduces to the classical Darcy's law. In what follows, we cannot give any detailed analysis of all the regions and interfaces shown in Figure 1. Instead, we select a few to highlight the principles.

\section{AN EXAMPLE OF FIELD EQUATIONS: TEMPERATE ICE SATURATED WITH WATER}

We treat saturated temperate ice as an incompressible, binary mixture of ice and water, and assume that ice and water have a common temperature at the melting point. This means that two balance laws of mass and momentum must be formulated, but only one energy relation. This energy relation cannot be an evolution equation for the temperature but must describe how much the internal energy changes as the result of phase changes. Essentially it yields the equation for the melting rate. For earlier treatments of temperate ice, see Hutter (1982) and Fowler (1984).

We assume both water and ice to be incompressible. Porosity, $n_{\mathrm{w}}$, and the true densities of the constituents, $\hat{\rho}_{\mathrm{W}}$ and $\hat{\rho}_{\mathrm{i}}$, are then related to the partial densities of the constituent by

$$
\begin{gathered}
\rho_{\mathrm{w}}=n_{\mathrm{w}} \hat{\rho}_{\mathrm{w}} \\
\rho_{\mathrm{i}}=\left(1-n_{\mathrm{w}}\right) \hat{\rho}_{\mathrm{i}}
\end{gathered}
$$

The porosity is usually small. With Equation (7), the mass-balance statements for the constituents water and ice become

$$
\begin{gathered}
\frac{\partial n_{\mathrm{w}}}{\partial t}+\left[n_{\mathrm{w}} v_{w}^{i}\right]_{, i}=\frac{\mathrm{c}_{\mathrm{w}}}{\hat{\rho}_{\mathrm{w}}} \\
-\frac{\partial n_{\mathrm{w}}}{\partial t}+\left[\left(1-n_{\mathrm{w}}\right) v_{\mathrm{i}}{ }^{i}\right]_{, i}=-\frac{\mathrm{c}_{\mathrm{w}}}{\hat{\rho}_{\mathrm{i}}}=-\frac{1}{\lambda} \frac{\mathrm{c}_{\mathrm{w}}}{\hat{\rho}_{\mathrm{w}}}
\end{gathered}
$$

where $\lambda=\hat{\rho}_{\mathrm{i}} / \hat{\rho}_{\mathrm{w}}=0.917$. In the momentum balances we ignore acceleration terms, introduce the peculiar stresses according to Equation (5) (example 3) and employ Darcy's law according to Equation (6) (example 5). Under such conditions the force balances of ice and water become

$$
\begin{gathered}
t_{\mathrm{i}, j}^{i j^{\prime}}-\delta^{i j}\left[\left(1-n_{\mathrm{w}}\right) p_{\mathrm{w}}\right]_{, j}+\left(1-n_{\mathrm{w}}\right) \hat{\rho}_{\mathrm{i}} g^{i}+ \\
+\frac{\mu}{k^{*}} n_{\mathrm{w}}^{2}\left[v_{\mathrm{w}}^{i}-v_{\mathrm{i}}^{i}\right]-\mathrm{c}_{\mathrm{w}} v_{\mathrm{w}}^{i}=0 \\
t_{\mathrm{w}, j}^{i j^{\prime}}-\delta^{i j}\left(n_{\mathrm{w}} p_{\mathrm{w}}\right)_{, j}+n_{\mathrm{w}} \hat{\rho}_{\mathrm{w}} g^{i}-\frac{\mu}{k^{*}} n_{\mathrm{w}}^{2}\left[v_{\mathrm{w}}^{i}-v_{\mathrm{i}}^{i}\right]+ \\
+\mathrm{c}_{\mathrm{w}} v_{\mathrm{w}}^{i}=0
\end{gathered}
$$

We still must formulate constitutive relations for the peculiar stresses. We suppose that the interstitial water is an ideal fluid, so that

$$
t_{\mathrm{w}}^{i j^{\prime}}=0
$$

In the case of ice, caution is advisable; the reason is that incompressibility of the mixture does not imply incompressibility of the ice, because the water content may vary due to the variation in the melting rate within the temperate-ice region. So Glen's flow law, for instance, 
cannot be, taken over blindly. Consequently, the constitutive stress $t_{\mathrm{i}}{ }^{i j^{\prime}}$ may have a non-vanishing isotropic part, $p_{\mathrm{i}}$, so that

$$
\begin{gathered}
t_{\mathrm{i}}^{i j^{\prime}}=-p_{\mathrm{i}} \delta^{i j}+\bar{t}_{\mathrm{i}}^{i j}=0 \\
\bar{t}_{\mathrm{i}}^{i j} \delta_{i j}=0
\end{gathered}
$$

An extension of Glen's flow law may then take the form

$$
\begin{gathered}
D_{\mathrm{i}}^{i j}-\frac{1}{3} D_{\mathrm{i} k}^{k} \delta^{i j}=A\left(x_{\mathrm{w}}\right) f\left(\bar{t}_{\mathrm{i}}^{\mathrm{II}}\right) \bar{t}_{\mathrm{i}}^{i j} \\
D_{\mathrm{i} k}^{k}=-3 g\left(p_{\mathrm{i}}\right) p_{\mathrm{i}}
\end{gathered}
$$

where

$$
\begin{gathered}
D_{\mathrm{i} j}^{i}=\frac{1}{2}\left(v_{\mathrm{i}, j}^{i}+v_{\mathrm{i}, i}^{j}\right) \\
\bar{t}_{\mathrm{i}}^{\mathrm{II}}=\frac{1}{2} \bar{t}_{\mathrm{i}}^{i j} \bar{t}_{\mathrm{i} i j}
\end{gathered}
$$

$A$ is a rate factor, which depends on the moisture content; $f\left(\bar{t}_{\mathrm{i}}^{\mathrm{II}}\right)$ is a creep-response function, assumed to be a function of the second stress-deviator invariant of constituent ice, and $g\left(p_{\mathrm{i}}\right)$ is a bulk viscosity.

Adding the relations (9) yields

$t_{i, j}^{i j}-\delta^{i j} p_{\mathrm{w}, j}+\rho g^{i}=0,\left(\rho=n_{\mathrm{w}} \hat{\rho}_{\mathrm{w}}+\left(1-n_{\mathrm{w}}\right) \hat{\rho}_{\mathrm{i}}\right)$

Alternatively, from the second part of Equation (9) we may deduce

$$
v_{\mathrm{F}}^{i}=\frac{k^{*}}{\mu}\left[-\delta^{i j} p_{\mathrm{w}, j}+\hat{\rho}_{\mathrm{w}} g^{i}\right]+\frac{k^{*}}{\mu n_{\mathrm{w}}} \mathrm{c}_{\mathrm{w}} v_{\mathrm{w}}^{i}
$$

where gradients $n_{\mathrm{w}, i}$ have been ignored. Introducing the gravitational potential such that

$$
g^{i}=-g \delta^{i j} \Phi, j
$$

and defining the piezometric head as

$$
h=\frac{p_{\mathrm{w}}}{\hat{\rho}_{\mathrm{w}} g}+\Phi
$$

Equation (13) can be written as

$$
\begin{gathered}
v_{\mathrm{F}}^{i}=-k\left[\delta^{i j} h, j-\frac{1}{\hat{\rho}_{\mathrm{w}} g n_{\mathrm{w}}} \mathrm{c}_{\mathrm{w}} v_{\mathrm{w}}^{i}\right] \\
k=\frac{\hat{\rho}_{\mathrm{w}} g k^{*}}{\mu}
\end{gathered}
$$

where $k$ is Darcy's permeability. Note how Equation (17) is modified relative to its classical expression when no melting takes place. Instead of relation (9) we may thus use the alternative relations (13) and (17). These equations more closely resemble the structure of the equations of soil mechanics.

Let us see what we can infer from energy considerations. In terms of our binary mixture concept, we have

$$
\rho \varepsilon=\rho_{\mathrm{w}} \varepsilon_{\mathrm{w}}+\rho_{\mathrm{i}} \varepsilon_{\mathrm{i}}+\frac{1}{2} \sum_{\alpha} \rho_{\alpha} u_{\alpha}^{2}
$$

If we write $\varepsilon_{\mathrm{w}}=c_{\mathrm{w}} T_{\mathrm{w}}$ and $\varepsilon_{\mathrm{i}}=c_{\mathrm{i}} T_{\mathrm{i}}$, where $c_{\mathrm{w}}$ and $c_{\mathrm{i}}$ are the specific heats of ice and water respectively, we obtain

$$
\rho \varepsilon=n_{\mathrm{w}} \hat{\rho}_{\mathrm{w}} c_{\mathrm{w}} T_{\mathrm{w}}+\left(1-n_{\mathrm{w}}\right) \hat{\rho}_{\mathrm{i}} c_{\mathrm{i}} T_{\mathrm{i}}+\frac{1}{2} \sum_{\alpha} \rho_{\alpha} u_{\alpha}^{2}
$$

Now since $T_{\mathrm{w}}=T_{\mathrm{i}}=T_{\mathrm{M}}$ (melting temperature) and $c_{\mathrm{w}} T_{\mathrm{M}}=c_{\mathrm{i}} T_{\mathrm{M}}+L$, where $L$ is the change of internal energy due to fusion, we have

$$
\varepsilon=c_{\mathrm{i}} T_{\mathrm{M}}+x_{\mathrm{w}} L+\frac{1}{2} \sum_{\alpha} \rho_{\alpha} u_{\alpha}^{2}
$$

The last term is usually ignored. If we discount heat conduction the energy equation becomes

$$
\rho \dot{\varepsilon}=t^{i j} D_{i j}
$$

where $t^{i j}$ are the components of the mixture stress, and $D_{i j}$ are the components of the barycentric stretching tensor. With Equations (12) and (18), we have

$$
\begin{gathered}
\rho L \dot{x}_{\mathrm{w}}=3\left(p_{\mathrm{w}}+p_{\mathrm{i}}\right)\left[x_{\mathrm{i}} g\left(p_{\mathrm{i}}\right) p_{\mathrm{i}}-\frac{1}{3} x_{\mathrm{w}} D_{\mathrm{w} k}^{k}\right]+ \\
+x_{\mathrm{w}} \bar{l}^{i j} D_{\mathrm{w} i j}+2 x_{\mathrm{i}} A\left(x_{\mathrm{w}}\right) f\left[\bar{t}_{\mathrm{i}}^{\mathrm{II}}\right) \bar{t}_{\mathrm{i}}^{\mathrm{II}}
\end{gathered}
$$

The intention is that the rate of water production, $c_{w}$, is implicitly described by Equation (20). It may be mentioned in passing that Hutter (1982) used the relation $\mathrm{c}_{\mathrm{w}}=t^{i j} D_{i j} / L$.

\section{AN EXAMPLE OF JUMP CONDITIONS: COLD- TEMPERATE TRANSITION SURFACE}

This is a singular surface where phase changes may take place. On the cold-ice side the material is a viscous fluid, on the temperate-ice side the body is a mixture of two fluids - one viscous, the other ideal. We postulate that the temperature does not experience a jump and that the tangential ice velocity is continuous, viz.

$$
\begin{gathered}
{[T]=0} \\
{\left[v_{\mathrm{i}}{ }^{i}-\left(v_{\mathrm{i}}{ }^{j} \widehat{e}_{j}\right) \hat{e}^{i}\right]=0}
\end{gathered}
$$

Since there is no water on the cold side one may request that $v_{\mathrm{w}}^{i+}=0$, but this would require the water phase to be viscous, which we have not assumed.

The surface consists of two separate parts: cold ice adjoining non-saturated, temperate ice, and cold ice adjoining saturated, temperate ice. We treat both at once, only we must make the distinction that in the saturated region $\rho_{\mathrm{w}}=n_{\mathrm{w}} \hat{\rho}_{\mathrm{w}}$, whereas this is not so in the non-saturated region. Let

$$
F_{\mathrm{c}}(x, t)=z-z_{\mathrm{c}}(x, y, t)=0
$$

denote the defining equation for this surface. Then (Fig. 2)

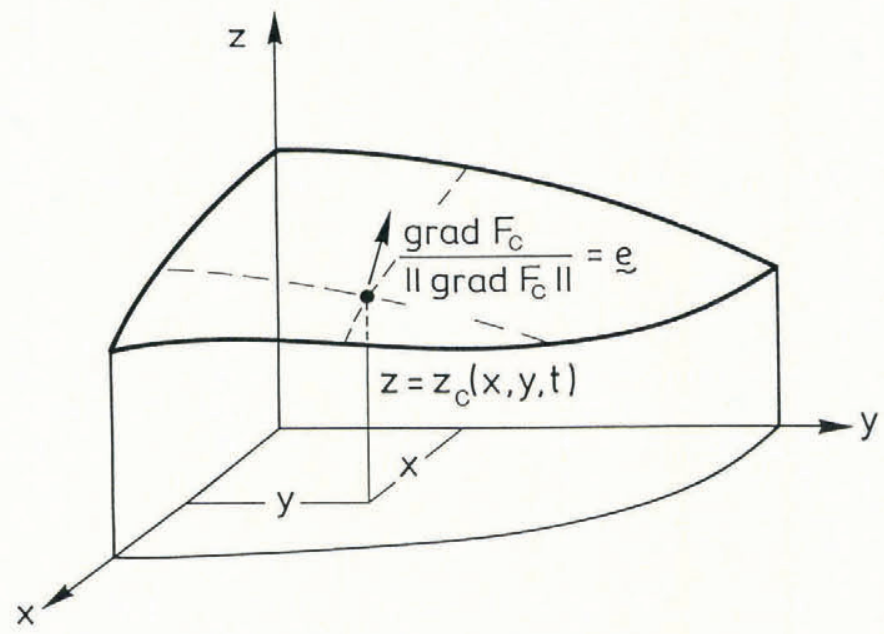

Fig. 2. Illustration of the free surface of an ice sheet, the kinematic equation (Equation (22)) 


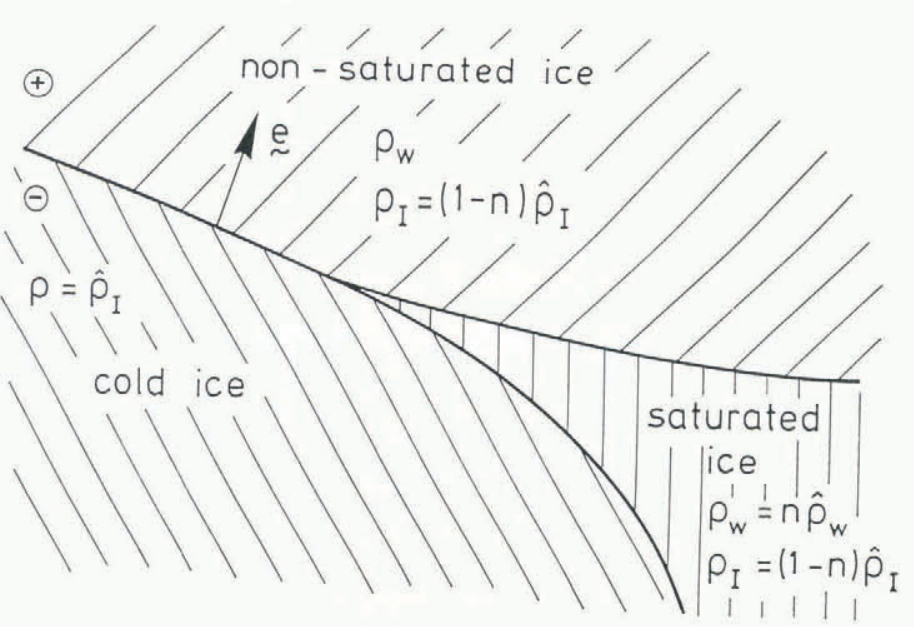

Fig. 3. Cold-temperate transition surface and phreatic surface separating cold ice, non-saturated and saturated temperate ice.

$$
\begin{gathered}
\frac{\partial z_{\mathrm{c}}}{\partial t}+\frac{\partial z_{\mathrm{c}}}{\partial x} v_{\mathrm{i}}{ }^{x-}+\frac{\partial z_{\mathrm{c}}}{\partial y} v_{\mathrm{i}}{ }^{y-}-v_{\mathrm{i}}{ }^{z-}=N_{\mathrm{c}} a_{\perp}^{\mathrm{c}} \\
N_{\mathrm{c}}=\left\{1+\left[\frac{\partial z_{\mathrm{c}}}{\partial x}\right)^{2}+\left[\frac{\partial z_{\mathrm{c}}}{\partial y}\right)^{2}\right\}^{\frac{1}{2}} \\
a_{\perp}^{\mathrm{c}}=-\left(v_{\mathrm{i}}{ }^{i}-w^{i}\right) \hat{e}_{i}
\end{gathered}
$$

The first part of Equation (22) is the evolution equation, in which $v_{\mathrm{i}}^{-}$is the velocity of the cold ice (Fig. 3), $w$ is the singular surface velocity, and $a_{\perp}^{c}$ is the freezing or melting rate perpendicular to the surface. In Equation (22) and in Figure 3 it is positive as a freezing rate. The jump conditions of mass are expressible as

$$
\begin{gathered}
\rho_{\mathrm{i}}^{+}\left[v_{\mathrm{i}}{ }^{i}\right] \hat{e}_{i}=\left[\rho_{\mathrm{i}}\right] a_{\perp}^{\mathrm{c}} \\
a_{\perp}^{\mathrm{c}}=\left[v_{\mathrm{w}}^{i+}-v_{\mathrm{i}}{ }^{i-}\right] \cdot \hat{e}_{i}
\end{gathered}
$$

Equation (23) relates the jump of the normal component of the ice velocity to the freezing rate; Equation (24) relates the freezing rate to the water velocity on the temperate side. When $a_{\perp}^{c}=0$, the normal component of the ice velocity is continuous and the water velocity at the interface is tangential to that of the ice. Both properties are to be expected.

Next, consider the jump conditions of momentum. For the constituent ice we obtain

$$
\rho_{\mathrm{i}}^{-} a_{\perp}^{\mathrm{c}}\left[v_{\mathrm{i}}^{i}\right]-\left[\left(1-n_{\mathrm{w}}\right) p_{\mathrm{w}} \hat{e}^{i}-t_{\mathrm{i}}^{i j^{\prime}} \hat{e}_{j}\right]=0
$$

The first term can be ignored; then Equation (25) requires continuity of the normal and shear stresses.

The jump conditions of momentum for the mixture as a whole can be shown to have the form

$$
n_{\mathrm{w}}^{+} p_{\mathrm{w}}^{+} \hat{e}^{i}=x_{\mathrm{w}}^{+}\left(v_{\mathrm{w}}^{i+}-v_{\mathrm{i}}^{i+}\right) \rho_{\mathrm{i}}^{-} a_{\perp}^{\mathrm{c}}
$$

It is important that we do not ignore $a_{\perp}^{c}$ in Equation (26); if we do, we will require that either $n_{\mathrm{w}}^{+}=0$ or $p_{\mathrm{W}}^{+}=0$, both of which are not reasonable. In Equation (25) we may, however, without hesitation drop the first term. Thus the momentum-jump conditions imply approximately

$$
\begin{gathered}
{\left[\left(1-n_{\mathrm{w}}\right) p_{\mathrm{w}} \hat{e}^{i}-t_{\mathrm{i}}^{i j} \hat{e}_{j}\right]=0} \\
n_{\mathrm{w}}^{+} p_{\mathrm{w}}^{+} \hat{e}^{i}=\left[\left[x_{\mathrm{i}} v_{\mathrm{i}}^{i}\right]-x_{\mathrm{w}}^{+} v_{\mathrm{w}}^{i+}\right] \rho_{\mathrm{i}}^{-} a_{\perp}^{\mathrm{c}}
\end{gathered}
$$

Next consider the entropy balance. With the first part of Equation (21) it may be written as

$$
\left[q^{i} \hat{e}_{i}\right]=\hat{\rho}_{\mathrm{i}} a_{\perp}^{\mathrm{c}} T[n]
$$

Since $n^{+}=x_{\mathrm{i}} n_{\mathrm{i}}+x_{\mathrm{w}} \eta_{\mathrm{w}}$, this becomes

$$
\begin{gathered}
{\left[q^{i} \hat{e}_{i}\right]=\frac{\hat{\rho}_{\mathrm{i}}}{1+\left(1-n_{\mathrm{w}}\right) \rho_{\mathrm{w}} / \hat{\rho}_{\mathrm{i}}} L a_{\perp}^{\mathrm{c}}} \\
L=T\left(n_{\mathrm{w}}-n_{\mathrm{i}}\right)
\end{gathered}
$$

where $L$ is the latent heat of fusion. This last relation is extremely important, as it relates the kink in the temperature at the cold-temperate transition surface to the melting rate.

\section{CONCLUDING REMARKS}

The above presentation only gives indications of the theoretical complexities one encounters when models that include the features shown in Figure 1 are considered. For detailed analyses, the reader may consult the papers of Hutter and Engelhardt (1988), and Hutter and Engelhardt (in preparation). It is obvious that computations performed with these models are still a great deal ahead of us.

\section{ACKNOWLEDGEMENT}

We thank Bob Svendsen for his help with the preparation of this manuscript.

\section{REFERENCES}

Fowler, A.C. 1984. On the transport of moisture in polythermal glaciers. Geophys. Astrophys. Fluid Dyn., 28, 99-140.

Hutter, K. 1982. A mathematical model of polythermal glaciers and ice sheets. Geophys. Astrophys. Fluid Dyn., 21, 201-224.

Hutter, K. 1983. Theoretical glaciology; material science of ice and the mechanics of glaciers and ice sheets. Dordrecht, etc., D. Reidel Publishing Company.

Hutter, K., and H. Engelhardt. 1988. How useful is continuum thermodynamics to formulate concepts of ice sheet dynamics? Eidg. Tech. Hochschule, Zürich. Versuchsanst. Wasserbau, Hydrol. Glaziol. Mitt., 94. 